\title{
On the Link Adaptation and User Scheduling with HARQ in the Presence of Inter-Cell Interference
}

\author{
Su Min Kim ${ }^{\dagger}$, Bang Chul Jung ${ }^{\ddagger}$, and Dan Keun Sung $§$ \\ ${ }^{\dagger}$ ACCESS Linneaus Centre, Royal Institute of Technology (KTH), Stockholm, Sweden \\ $\ddagger$ Department of Information and Communication Engineering, Gyeongsang National University, Tongyeong, Korea \\ $\S$ Department of Electrical Engineering, KAIST, Daejeon, Korea \\ E-mail: sumin@kth.se, bcjung@gnu.ac.kr, dksung@ee.kaist.ac.kr
}

\begin{abstract}
In this paper, we investigate both link adaptation and user scheduling for an HARQ-based multi-user system in downlink multi-cell environments with inter-cell interference (ICI). First, we analyze two approximation methods on the ICI terms for optimal rate selection. One is a conventional well-known Gaussian approximation (GA) and the other is our proposed identical path-loss approximation (IPLA). Thereafter, we propose a baseline procedure for both the link adaptation and user scheduling. Then, we introduce three conventional policies and our proposed IPLA-based policy. Finally, through system-level simulations, the proposed IPLA-based policy is evaluated in terms of system throughput. Numerical results show that the traditional GA is not accurate any more in the HARQ-based multi-user system with some dominant ICIs. Instead of the GA, our proposed IPLA-based policy achieves more accurate rate selection and higher system performance.
\end{abstract}

\section{INTRODUCTION}

To improve both link reliability and resource efficiency, a hybrid automatic repeat request (HARQ) scheme has been proposed in physical layer. In medium access control (MAC) layer, dynamic link adaptation and user scheduling techniques have been developed in order to enhance the resource efficiency in a single point-to-point and multi-user environments, respectively. Both techniques significantly enhance the resource efficiency using channel status information (CSI) at the transmitter. So far, there are many previous work related to link adaptation considering the HARQ schemes in various fading channel models [1], [2] and user scheduling considering the HARQ schemes in multi-user environments [3], [4].

On the other hand, in multi-cell based cellular networks, interference from other cells is another key factor to determine the system performance. In CDMA-based cellular networks, inter-cell interference (ICI) is regarded as one of other interference sources which are added to intra-cell interference caused by other users in their own cell since it is basically an interference-limited system based on spectrum spreading (i.e., interference averaging). However, in OFDM-based cellular networks, the ICI caused by data transmissions using the same resource area in other cells significantly affects the desired signal because there is no effect of interference averaging. That is, there exist some dominant interferers caused by adjacent neighboring cells. Therefore, for efficient link adaptation and user scheduling, the ICI also needs to be carefully managed in OFDM-based multi-cell networks.
Recently, there have been some studies on HARQ-based multi-user systems in the presence of interference. Narasimhan [5] analyzed the total user throughput in a two-user interference channel through receiver cooperation. Denic [6] proposed a robust HARQ-incremental redundancy (IR) coding scheme in the presence of unknown interference such as jamming. In MIMO-based HARQ systems, various HARQ schemes were proposed taking into account inter-carrier interference and inter-antenna interference [7], [8]. Rácz et al. [9] studied the gain of an inter-cell interference coordination (ICIC) mechanism in the uplink 3GPP LTE system. Although they investigated system-level aspects for ICIC in a multi-cell environment, optimal rate adaptation and multiuser scheduling issues were not taken into account. ShiraniMehr et al. [10] proposed an optimal scheduling and ARQ scheme with a game theoretic approach in a multi-user (MU)MIMO system with the ICI. They attempted to solve a joint operation problem of scheduling, MU-MIMO beamforming, and $A R Q$ in a distributed manner. Even if they studied a tradeoff between system throughput and transmission delay, dynamic link adaptation was not considered.

The rest of this paper is organized as follows. In Section II, we introduce a system model. In Section III, we investigate link adaptation schemes based on the proposed IPLA and the conventional GA. In Section IV, we present a baseline procedure for user scheduling including the link adaptation and introduce various policies. In Section V, we show the performance of the proposed IPLA policy, compared to the three conventional policies, through system-level simulations. Finally, we present conclusive remarks in Section VI.

\section{SySTEM MODEL}

Fig. 1 shows a system model considered in this paper. We take into account a downlink SU-MISO system. There are $(K+1)$ base stations (BSs) with $M$ antennas and $N$ users with a single antenna in each cell. Here, a BS in home cell is indicated by superscript (0) and BSs in other cells are indicated by superscript $(k), k=\{1, \ldots, K\}$. At a certain moment, each BS schedules a user within its own cell and transmit data by means of random beamforming (RBF) [11].

A scheduled user in each cell receives a desired signal, which is multiplied by a beamforming vector and a channel vector, from its own BS. At this moment, the scheduled user 


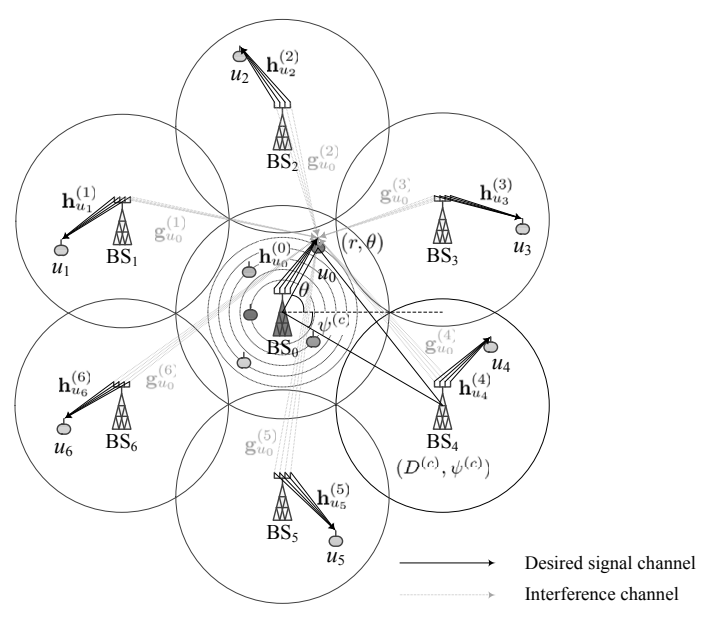

Fig. 1. System Model

also experiences ICI from the BSs in other $K$ cells. Without loss of generality, we just focus on a user selected in the home cell since all the BSs and users operate in the same manner. The received signal of the scheduled user in the home cell (i.e., $k=0$ ) is expressed as

$$
y_{u_{0}}^{(0)}=\mathbf{h}_{u_{0}}^{(0)} \mathbf{v}_{u_{0}}^{(0)} x_{u_{0}}^{(0)}+\sum_{k=1}^{K} \mathbf{g}_{u_{0}}^{(k)} \mathbf{v}_{u_{k}}^{(k)} x_{u_{k}}^{(k)}+n_{u_{0}}^{(0)},
$$

where $u_{k}$ denotes the scheduled user index in the $k$-th cell, $\mathbf{h}_{u_{0}}^{(0)}$ and $\mathbf{g}_{u_{0}}^{(k)}$ denote the channel vectors for the desired signal from the home cell and interference signal from the $k$-th cell, respectively, $\mathbf{v}_{u_{k}}^{(k)}$ indicates the RBF vector for user $u_{k}$ in the $k$-th cell and $x_{u_{k}}^{(k)}$ denotes the source data symbol of user $u_{k}$, and $n_{u_{0}}^{(0)}$ denotes the additive white Gaussian noise (AWGN), i.e., $n_{u_{0}}^{(0)} \sim \mathcal{C N}\left(0, N_{0}\right)$.

In Eq. (1), $\mathbf{h}_{u_{0}}^{(0)}$ and $\mathbf{g}_{u_{0}}^{(k)}$ denote the MISO channel vectors including large-scale and small-scale fading elements and they are expressed as follows:

$$
\begin{aligned}
& \mathbf{h}_{u_{0}}^{(0)}=\left[\sqrt{L_{u_{0}}^{(0)}} h_{u_{0}, 1}^{(0)}, \sqrt{L_{u_{0}}^{(0)}} h_{u_{0}, 2}^{(0)}, \cdots, \sqrt{L_{u_{0}}^{(0)}} h_{u_{0}, M}^{(0)}\right], \\
& \mathbf{g}_{u_{0}}^{(k)}=\left[\sqrt{L_{u_{0}}^{(k)}} g_{u_{0}, 1}^{(k)}, \sqrt{L_{u_{0}}^{(k)}} g_{u_{0}, 2}^{(k)}, \cdots, \sqrt{L_{u_{0}}^{(k)}} g_{u_{0}, M}^{(k)}\right],
\end{aligned}
$$

where $h_{u_{0}, m}^{(0)}$ and $g_{u_{0}, m}^{(k)}$ denote the small-scale fading signal term of user $u_{0}$ from the $m$-th antenna of the home BS and the $k$-th $\mathrm{BS}$, respectively, and they are complex Gaussian random variables with zero mean and unit variance. We assume a slowly varying channel condition and, thus, $h_{u_{0}, m}^{(0)}$ and $g_{u_{0}, m}^{(k)}$ are quasi-static during one HARQ retransmission process. $L_{u_{0}}^{(0)}$ and $L_{u_{0}}^{(k)}$ denote the large-scale fading power term of user $u_{0}$ from the home $\mathrm{BS}$ and the $k$-th $\mathrm{BS}$, respectively. In this paper, we neglect a shadowing factor and just consider path-loss as a large-scale fading. Thus, $L_{u_{0}}^{(k)}$ is expressed as $10^{-\frac{\mathrm{PL}}{10}} \cdot\left(d_{0} / d_{u_{0}}^{(k)}\right)^{\alpha},(k=0, \ldots, K)$ where $\mathrm{PL}_{0}$ denotes the path-loss in $\mathrm{dB}$ at reference distance $d_{0}, \alpha$ denotes the pathloss exponent, and $d_{u_{0}}^{(k)}$ represents the distance between user $u_{0}$ and the $k$-th BS. When user and BS locations in the home cell is expressed as $(r, \theta)$ and $\left(D^{(k)}, \psi^{(k)}\right)$, respectively, $d_{u_{0}}^{(k)}$ can be calculated by $\sqrt{r^{2}+\left(D^{(k)}\right)^{2}-2 r D^{(k)} \cos \left(\theta-\psi^{(k)}\right)}$, $(k=0, \ldots, K)$ where $D^{(0)}=0$ (i.e., $\left.d_{u_{0}}^{(0)}=r\right)$.

As mentioned before, we consider the RBF scheme in this paper. Therefore, as shown in [11], the beamforming vectors in Eq. (1) are expressed as $\mathbf{v}_{u_{k}}^{(k)}=\left[v_{u_{k}, 1}^{(k)}, v_{u_{k}, 2}^{(k)}, \ldots, v_{u_{k}, M}^{(k)}\right]^{T}$, $(k=0, \ldots, K)$ where $[\cdot]^{T}$ denotes the transpose of a vector, $v_{u_{k}, m}^{(k)}=\sqrt{a_{m}} e^{j \theta_{m}}$ where $a_{m} \in[0,1], \theta_{m} \sim$ Uniform $[-\pi, \pi]$, and $\left\|\mathbf{v}_{u_{k}, m}^{(k)}\right\|^{2}=\sum_{m=1}^{M} a_{m}=1$. From a property of the RBF scheme, the sum of ICI terms in Eq. (1) is derived by

$$
\begin{aligned}
\mathcal{I} & \triangleq \sum_{k=1}^{K} \mathbf{g}_{u_{0}}^{(k)} \mathbf{v}_{u_{k}}^{(k)} x_{u_{k}}^{(k)} \\
& =\sum_{k=1}^{K} \sqrt{L_{u_{0}}^{(k)}}(\underbrace{\sqrt{\alpha_{1}} e^{j \theta_{1}} g_{u_{0}, 1}^{(k)}+\cdots+\sqrt{\alpha_{M}} e^{j \theta_{M}} g_{u_{0}, M}^{(k)}}_{\sim \mathcal{C N}(0,1)}) x_{u_{k}}^{(k)} \\
& =\sum_{k=1}^{K} \sqrt{L_{u_{0}}^{(k)}} w_{u_{0}}^{(k)} x_{u_{k}}^{(k)}
\end{aligned}
$$

where $w_{u_{0}}^{(k)} \sim \mathcal{C N}(0,1)$ and the third equality comes from an isotropic property of complex Gaussian random variable.

Consequently, the received SINR of the scheduled user in the home cell is written as

$$
\gamma=\frac{s}{\mathcal{X}+1 / \rho}
$$

where $s=\left\|\mathbf{h}_{u_{0}}^{(0)} \mathbf{v}_{u_{0}}^{(0)}\right\|^{2}=L_{u_{0}}^{(0)}\left|w_{u_{0}}^{(0)}\right|^{2}, \mathcal{X}=|\mathcal{I}|^{2} / P_{x}=$ $\sum_{k=1}^{K} L_{u_{0}}^{(k)}\left|w_{u_{0}}^{(k)}\right|^{2}$, and $\rho=\frac{P_{x}}{N_{0}}$ where $\mathbb{E}\left[\left|x_{u_{k}}^{(k)}\right|^{2}\right]=P_{x}$ for $k=\{0, \ldots, K\}$. As assumed before, $L_{u_{0}}^{(0)}$ is a known constant based on user location information at the home BS and we further assume that the effective channel power gain of $u_{0}$, $\left|w_{u_{0}}^{(0)}\right|^{2}$, is perfectly known at the transmitter. It is reasonable because the home BS knows the RBF vector for user $u_{0}$ in advance and we also consider a quasi-static channel condition, in which it is possible to estimate the effective channel power gain perfectly, for desired signal channels. Hence, the desired power term $s$ is known as a constant at the transmitter. Note that the inverse of transmit SNR term is normally negligible in interference-limited regime (i.e., high SNR regime).

If we consider an HARQ-CC scheme, the effective SINR after HARQ-CC combining at the $n$-th transmission is important and is expressed as

$$
\gamma(n)=\sum_{i=1}^{n} \gamma_{i}=\sum_{i=1}^{n} \frac{s}{\mathcal{X}_{i}+1 / \rho}
$$

where $\mathcal{X}_{i}$ denotes the ICI channel power at the $i$-th transmission and is expressed as $\mathcal{X}_{i}=\sum_{k=1}^{K} L_{u_{0}}^{(k)}\left|w_{u_{0}}^{(k)}\left(t_{i}\right)\right|^{2}$ in which $t_{i}$ indicates the time slot index of the $i$-th transmission. Since the RBF vector is kept during retransmissions and the desired signal channel is quasi-static, $s$ is an identical value for every transmission. However, although interference channel condition $\mathbf{g}_{u_{0}}^{(k)}$ is also quasi-static, beamforming vectors in other cells are independently varying according to scheduling decision in other cells. Therefore, the ICI channel power terms are independently varying for every transmission. 


\section{Link AdAPTATION With HARQ-CC IN THE PRESENCE OF INTER-CELL INTERFERENCE}

In this section, we first mathematically analyze the distribution of the effective SINR and delay-limited throughput (DLT) which represents an expected throughput under a given maximum number of transmissions [1], [2]. Then, we propose an identical path-loss approximation (IPLA) scheme on the ICI terms in the effective SINR model. We also investigate the well-known conventional Gaussian approximation (GA) on the ICI plus noise terms through the same mathematical framework. For both of them, we provide optimal rate selection schemes to maximize the DLT. We start to derive the distribution of the effective SINR based on numerical inversion of characteristic function (CF) from the following theorem.

Theorem 1 (Inversion formula of Gil-Pelaez [12]): Let $\phi(t)=\int_{-\infty}^{\infty} e^{j t x} d F(x)$ be a CF of the one-dimensional distribution function $F(x)$. For $x$ being the continuity point of the distribution, the following inversion formula holds true:

$$
F(x)=\frac{1}{2}-\frac{1}{\pi} \int_{0}^{\infty} \mathcal{I} m\left(\frac{e^{-j t x} \phi(t)}{t}\right) d t .
$$

Proof: Refer to [12]

By using Theorm 1, if we know the CF of the effective SINR after the $n$-th (re)transmission which is denoted by $\phi_{\gamma(n)}(t)$, we can obtain the cumulative distribution function (CDF), $F_{\gamma(n)}(x)$. We now assume the information-theoretic capacityachieving channel coding and continuous modulation schemes and then the outage probability after the $n$-th (re)transmission is defined as $P_{\text {out }}(n, R) \triangleq \operatorname{Pr}\left\{\log _{2}(1+\gamma(n))<R\right\}=$ $F_{\gamma(n)}\left(2^{R}-1\right)$ where $R$ denotes the required data rate. As shown in [2], the DLT is defined as

$$
S(R)=\sum_{i=1}^{N_{\max }} \frac{R}{i}\left[P_{\text {out }}(i-1, R)-P_{\text {out }}(i, R)\right],
$$

where $N_{\max }$ denotes the maximum number of transmissions. In a result, the DLT is rewritten as

$$
\begin{aligned}
& S(R)=\sum_{i=1}^{N_{\max }} \frac{R}{i \cdot \pi} \int_{0}^{\infty} {\left[\mathcal { I } m \left\{\frac{e^{-j t\left(2^{R}-1\right)}}{t}\right.\right.} \\
&\left.\left.\cdot\left(\phi_{\gamma(i)}(t)-\phi_{\gamma(i-1)}(t)\right)\right\}\right] d t .
\end{aligned}
$$

\section{A. Link Adaptation Based on the Proposed Identical Path-Loss Approximation (IPLA)}

Assuming the interference-limited regime (i.e., $\rho \gg 1$ ), the effective SINR after HARQ-CC combining is rewritten as

$$
\gamma(n) \approx \sum_{i=1}^{n} \frac{s}{\mathcal{X}_{i}}=\sum_{i=1}^{n} \frac{s}{\sum_{k=1}^{K} L_{u_{0}}^{(k)}\left|w_{u_{0}}^{(k)}\left(t_{i}\right)\right|^{2}},
$$

where $t_{i}$ denotes the time slot index of the $i$-th transmission. The sum of ICI terms in the denominator is a linear combination of weighted Gamma random variables. Thus, the distribution of the effective SINR has a complicated form and this is intractable. Therefore, we assume that all the path-loss terms from BSs are identical as an average value of them in Eq. (10) and then the effective SINR is approximated by

$$
\gamma(n) \approx \sum_{i=1}^{n} \tilde{\gamma}_{i}=\sum_{i=1}^{n} \frac{s}{\sum_{k=1}^{K} \bar{L}\left|w_{u_{0}}^{(k)}\left(t_{i}\right)\right|^{2}},
$$

where $\bar{L}=\frac{1}{K} \sum_{k=1}^{K} L_{u_{0}}^{(k)}$. Now, the sum of ICI terms becomes the sum of identical and independently distributed (i.i.d.) Gamma random variables and it also follows a Gamma distribution. After all, the approximated SINR at the $i$-th transmission, $\tilde{\gamma}_{i}$, follows an inverted Gamma distribution, i.e., $\tilde{\gamma}_{i} \sim \operatorname{Inv-\operatorname {Gamma}}(K, s / \bar{L})$. Hence, the CF of $\tilde{\gamma}_{i}$ is derived as follows: [13]

$$
\phi_{\tilde{\gamma}_{i}}(t)=\frac{2\left(-\frac{j s t}{L}\right)^{\frac{K}{2}}}{(K-1) !} K_{K}\left(\sqrt{-\frac{4 j s t}{\bar{L}}}\right),
$$

where $K_{\nu}(\cdot)$ denotes the modified Bessel function of the second kind. Since $\tilde{\gamma}_{i}$ 's are i.i.d. random variables, the $\mathrm{CF}$ of $\gamma(n)$ is obtained as

$$
\phi_{\gamma(n)}(t)=\prod_{i=1}^{n} \phi_{\tilde{\gamma}_{i}}(t)=\left[\frac{2\left(-\frac{j s t}{\bar{L}}\right)^{\frac{K}{2}}}{(K-1) !} K_{K}\left(\sqrt{-\frac{4 j s t}{\bar{L}}}\right)\right]^{n} .
$$

By substituting Eq. (13) for Eq. (9), the DLT of the proposed IPLA is obtained as

$$
\begin{aligned}
S_{\mathrm{IPLA}}(R)=\sum_{i=1}^{N_{\max }} \frac{R}{i \cdot \pi} \int_{0}^{\infty} & {\left[\mathcal { I } m \left\{\Psi_{\mathrm{IPLA}}(t ; i, R)\right.\right.} \\
& \left.\left.-\Psi_{\mathrm{IPLA}}(t ; i-1, R)\right\}\right] d t
\end{aligned}
$$

where $\Psi_{\text {IPLA }}(t ; i, R)=\frac{e^{-j t\left(2^{R}-1\right)}}{t}\left[\frac{2\left(-\frac{j s t}{L}\right)^{\frac{K}{2}}}{(K-1) !} K_{K}\left(\sqrt{-\frac{4 j s t}{L}}\right)\right]^{i}$. Finally, in order to maximize the DLT, the optimal source rate by the proposed IPLA is determined as follows:

$$
\begin{array}{r}
R_{\mathrm{IPLA}}^{*}=\underset{R \geq 0}{\operatorname{argmax}} \sum_{i=1}^{N_{\max }} \frac{R}{i \cdot \pi} \int_{0}^{\infty}\left[\mathcal { I } m \left\{\Psi_{\mathrm{IPLA}}(t ; i, R)\right.\right. \\
\left.\left.-\Psi_{\mathrm{IPLA}}(t ; i-1, R)\right\}\right] d t .
\end{array}
$$

\section{B. Link Adaptation through the Conventional Gaussian Ap- proximation (GA)}

Traditionally, the sum of ICI terms is widely approximated as a Gaussian distribution by the well-known central limit theorem (CLT) for even six interference components considering 7-cell structured cellular networks [14]. Through the GA on the ICI plus noise terms, the effective SINR after HARQ-CC combining is expressed as

$$
\gamma(n)=\sum_{i=1}^{n} \frac{s}{\mathcal{X}_{i}+1 / \rho} \approx \sum_{i=1}^{n} \tilde{\gamma}_{i}=\sum_{i=1}^{n} \frac{s}{\left|Z_{i}\right|^{2}}
$$

where $Z_{i}$ denotes a complex Gaussian random variable with zero mean and variance of the sum of ICI and noise powers, i.e., $Z_{i} \sim \mathcal{C N}\left(0, \sigma_{Z}^{2}\right)$ where $\sigma_{Z}^{2}=\sum_{k=1}^{K} L_{u_{0}}^{(k)}+1 / \rho$. Here, $\tilde{\gamma}_{i}$ 
is an inverted Gamma random variable with shape parameter 1 and scale parameter $s / \sigma_{Z}^{2}, \tilde{\gamma}_{i} \sim \operatorname{Inv-Gamma}\left(1, s / \sigma_{Z}^{2}\right) . \tilde{\gamma}_{i}$ obtained by the GA follows an identical distribution with different shape and scale parameters with that by the IPLA. Then, the CF of $\gamma(n)$ are derived as follows: [13]

$$
\phi_{\gamma(n)}(t)=\left[\sqrt{-\frac{4 j s t}{\sigma_{Z}^{2}}} K_{1}\left(\sqrt{-\frac{4 j s t}{\sigma_{Z}^{2}}}\right)\right]^{n} .
$$

By substituting Eq. (17) for Eq. (9), the DLT of the conventional GA is similarly obtained as

$$
\begin{aligned}
S_{\mathrm{GA}}(R)=\sum_{i=1}^{N_{\max }} \frac{R}{i \cdot \pi} \int_{0}^{\infty} & {\left[\mathcal { I } m \left\{\Psi_{\mathrm{GA}}(t ; i, R)\right.\right.} \\
& \left.\left.-\Psi_{\mathrm{GA}}(t ; i-1, R)\right\}\right] d t,
\end{aligned}
$$

where $\Psi_{\mathrm{GA}}(t ; i, R)=\frac{e^{-j t\left(2^{R}-1\right)}}{t}\left[\sqrt{-\frac{4 j s t}{\sigma_{Z}^{2}}} K_{1}\left(\sqrt{-\frac{4 j s t}{\sigma_{Z}^{2}}}\right)\right]^{k}$. Finally, to maximize the DLT, the optimal source rate through the conventional GA is determined as follows:

$$
\begin{aligned}
R_{\mathrm{GA}}^{*}=\underset{R \geq 0}{\operatorname{argmax}} \sum_{i=1}^{N_{\max }} \frac{R}{i \cdot \pi} \int_{0}^{\infty}\left[\mathcal { I } m \left\{\Psi_{G A}(t ; i, R)\right.\right. \\
\left.\left.-\Psi_{G A}(t ; i-1, R)\right\}\right] d t .
\end{aligned}
$$

\section{SCHEduling With HARQ-CC IN THE PREsence of INTER-CELL INTERFERENCE}

\section{A. A Baseline Procedure for Link Adaptation and Scheduling}

The baseline procedure consists of three main components: rate selection, effective rate mapping, and scheduler.

1) Rate Selection: The rate selection plays a role to determine an optimal source rate $R_{u}^{*}(t)$ for each user $u$ at initial transmission instance. In this paper, we consider rate selection schemes to maximize the DLT of each user as described in the previous subsection.

2) Effective Rate Mapping (ERM): The ERM determines an effective rate $R_{\mathrm{eff}, u}(t)$ for each user based on the optimal source rate $R_{u}^{*}(t)$ in order to adjust the scheduling priority of each user. By this ERM, the instantaneous rate $R_{u}(t)$ in the scheduler is replaced by the effective rate $R_{\mathrm{eff}, u}(t)$. After all, the scheduler selects a user with the highest value among utility values substituted for the effective rates.

3) Scheduler: The scheduler determines which user is the best at every scheduling instance. In this paper, we adopt a proportional fair (PF) scheduler to consider user fairness.

A baseline procedure is processed as follows:

(1) [Rate Selection]: Determine $R_{u}^{*}(t)$

(2) $[\mathbf{E R M}]$ : Determine $R_{\mathrm{eff}, u}\left(R_{u}^{*}(t)\right)$

(3) [Scheduler]: Determine $u^{*}=\underset{u \in \Pi}{\operatorname{argmax}} \frac{R_{\mathrm{eff}, u}\left(R_{u}^{*}(t)\right)}{T_{u}(t)}$

(4) [HARQ Transmission]

$-u^{*}$ transmits until successful transmission or maximum transmission limit.

- Go to (1) for all users after the end of the (re)transmissions of the scheduled user $u^{*}$.
B. Conventional and Proposed Link Adaptation and User Scheduling Policies

1) Conventional Policies:

- Instantaneous SINR Policy, $\mathcal{P}\left\{\mathrm{RA}-i\right.$-SINR, $\left.R_{\text {inst }}^{*}\right\}$ This policy is the simplest one where we consider the SINR value fed back from the receiver. The source rate of the $u$-th user is expressed as $R_{i \text {-SINR, } u}^{*}=$ $\log _{2}\left(1+\frac{s}{\sum_{k=1}^{K} L_{u_{0}}^{(k)}\left|w_{u_{0}}^{(k)}\left(t_{1}-\delta\right)\right|^{2}+1 / \rho}\right)$ where $\delta$ denotes the feedback delay and, thus, $\left|w_{u_{0}}^{(k)}\left(t_{1}-\delta\right)\right|^{2}$ represents the interference channel power gain from the $k$-th BS to user $u_{0}$ fed back from the receiver. Since this policy has just instantaneous information without consideration of HARQ retransmission, the Instantaneous Rate is employed as the ERM, i.e., $R_{\text {eff }, u}=R_{i \text {-SINR }, u}^{*}$.

- Average Interference Policy, $\mathcal{P}\left\{\mathrm{RA}-\mathrm{Avg}-\mathcal{X}, R_{\text {inst }}^{*}\right\}$

This policy just uses an average value for the sum of the ICI terms [8]. The source rate of the $u$-th user is expressed as $R_{\mathrm{Avg}-\mathcal{X}, u}^{*}=\log _{2}\left(1+\frac{s}{\mathcal{X}+1 / \rho}\right)$ where $\overline{\mathcal{X}}=$ $\mathbb{E}\left[\sum_{k=1}^{K} L_{u_{0}}^{(k)}\left|w_{u_{0}}^{(k)}\right|^{2}\right]=\sum_{k=1}^{K} L_{u_{0}}^{(k)}$. This policy has an identical source rate during retransmissions because of applying an average value for the ICI term. Thus, the Instantaneous Rate is also employed as the ERM, i.e., $R_{\mathrm{eff}, u}=R_{\mathrm{Avg}-\mathcal{X}, u}^{*}$.

- GA Policy, $\mathcal{P}\left\{\right.$ RA-GA, $\left.S_{G A}\left(R_{R A}^{*}\right)\right\}$

The GA policy is based on the link adaptation through the GA. Hence, the source rate of the $u$-th user is determined by Eq. (19). Since the GA policy considers an HARQ retransmission process by using the statistics of the ICI terms, the throughput-based ERM is employed as $R_{\mathrm{eff}, u}=S_{\mathrm{GA}}\left(R_{\mathrm{GA}}^{*}\right)$.

2) Proposed IPLA Policy, $\mathcal{P}\left\{R A-I P L A, S_{I P L A}\left(R_{I P L A}^{*}\right)\right\}$ : The proposed policy is based on the link adaptation by the IPLA. Thus, the source rate of the $u$-th user is determined by Eq. (15). Since the IPLA policy also considers an HARQ retransmission process by using the statistics of the ICI terms, the throughput-based ERM is employed as $R_{\mathrm{eff}, u}=S_{\mathrm{IPLA}}\left(R_{\mathrm{IPLA}}^{*}\right)$.

\section{Numerical Results}

In this section, we evaluate the performance of the proposed and conventional link adaptation and user scheduling policies through system-level simulations. We consider a 1-tier cellular network with six other cells where users are asymmetrically distributed in the home cell. The BS-to-BS distance is set to $1000 \mathrm{~m}$. We set the distances between users and the home BS to $r \in[150,200,250,300,400] \mathrm{m}$ and each element in the vector is equally set according to the number of users. For a single user case, it is set to be $250 \mathrm{~m}$. Additionally, the angles between users and the home BS are uniformly determined as $\theta=$ Uniform $[-\pi, \pi]$. For path-loss, we set $\mathrm{PL}_{0}$ to $37 \mathrm{~dB}$ at reference distance $d_{0}=1000 \mathrm{~m}$ and $\alpha=3$. In order to take into account an interference-limited situation, we set transmit SNR $\rho$ to $43 \mathrm{~dB}$ and finally $N_{\max }=4$. 


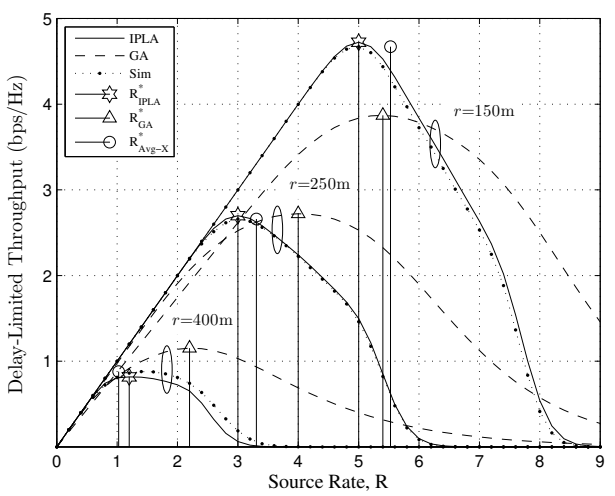

Fig. 2. Effect of User Distance on the Link Adaptation $(K=6, \theta=\pi / 2$, $\left.\alpha=3,\left|w_{u_{0}}^{(0)}\right|^{2}=1, N_{\max }=4\right)$

\section{A. Effects of User Distance on the Link Adaptation}

Fig. 2 shows the DLT for varying source rates $R$ in three different user distance values in a single link. As the distance decreases, higher DLT is achieved since the desired signal power increases. The DLT derived by the proposed IPLA has nearly the same values as that through the simulation for all distance values. However, optimal source rates based on the two conventional schemes exhibit some differences from the actual optimal-value in the simulations. The gap between the optimal source rates by the proposed IPLA and the GA becomes larger as the user distance increases, while the gap between the optimal source rates determined by the proposed IPLA policy and the average interference policy increases as the user distance decreases. Fundamentally, the two conventional schemes exhibit significant differences with respect to the optimal source rate for large SINR values. In a scheduling-based multi-user system, since a user with a large SINR value has more opportunities to be selected, the proposed IPLA can have a significant throughput gain.

\section{B. System Throughput}

Fig. 3 shows the system throughput for varying the number of users. The instantaneous SINR policy achieves the worst system throughput due to rather inaccurate estimation of the ICI term caused by the SINR feedback delay. The proposed IPLA policy always outperforms the conventional policies in the entire range of the number of users, while both the GA and average interference policies achieve almost identical system throughput. Note that although the GA policy exploits the statistics of the ICI term, it achieves similar system throughput to that of the average interference policy that just utilizes an average value of the ICI term. Moreover, it achieves rather smaller system throughput than the proposed IPLA policy which also exploits the equivalent statistics of the ICI term.

\section{CONCLUSION}

In this paper, we investigated both the link adaptation and user scheduling with HARQ in multi-cell environments with ICI. We first proposed an IPLA on the ICI terms for the link adaptation. Then, we proposed an IPLA-based scheduling policy. Our numerical results showed the proposed IPLA policy significantly outperforms the conventional GA policy

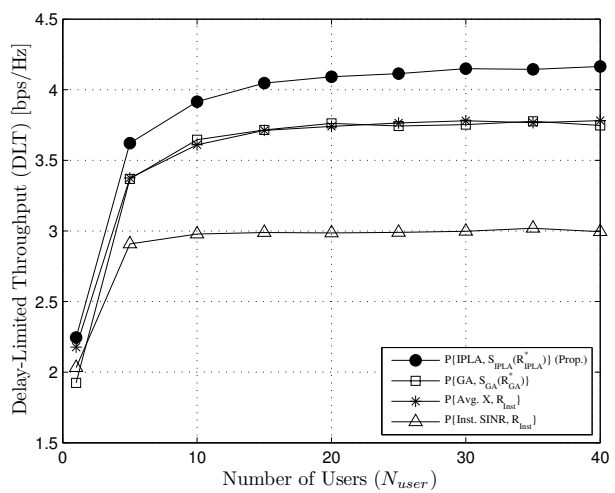

Fig. 3. System Throughput $(K=6, r \in[150,200,250,300,400] \mathrm{m}$, $\theta=$ Uniform $\left.[-\pi, \pi], \alpha=3, N_{\max }=4\right)$

in terms of system throughput, even if both of them exploit the same statistic of the ICI. Through this study, the traditional GA on the ICI term is not accurate any more in the HARQbased multi-user system with some dominant ICIs.

\section{ACKNOWLEDGMENT}

This research was supported by Basic Science Research Program through the National Research Foundation of Korea (NRF) funded by the Ministry of Education, Science and Technology (2010-0011140).

\section{REFERENCES}

[1] D. Kim, B. C. Jung, H. Lee, D. K. Sung, and H. Yoon, "Optimal modulation and coding scheme selection in cellular networks with hybrid-ARQ error control," IEEE Trans. Wireless Commun., vol. 7, no. 12, pp. 5195 - 5201, Dec. 2008.

[2] S. M. Kim, W. Choi, T. W. Ban, and D. K. Sung, "Optimal rate adaptation for hybrid ARQ in time-correlated Rayleigh fading channels," IEEE Trans. Wireless Commun., vol. 10, no. 3, pp. 968 - 979, Mar. 2011.

[3] H. Zheng and H. Viswanathan, "Optimizing the ARQ performance in downlink packet data systems with scheduling," IEEE Trans. Wireless Commun., vol. 4, no. 2, pp. 495 - 506, Mar. 2005.

[4] S. M. Kim, B. C. Jung, W. Choi, and D. K. Sung, "Effects of heterogeneous mobility on rate adaptation and user scheduling in cellular networks with HARQ," IEEE Trans. Veh. Technol., 2013 (To appear).

[5] R. Narasimhan, "Hybrid-ARQ interference channels with receiver cooperation," in Proc. IEEE ICC, May 2010.

[6] S. Z. Denic, "Robust incremental redundancy hybrid ARQ coding for channels with unknown interference," in Proc. IEEE ISIT, July 2011.

[7] R.-T. Juang, K.-Y. Lin, P. Ting, H.-P. Lin, and D.-B. Lin, "Enhanced chase combining HARQ with ICI and IAI mitigation for MIMO-OFDM systems," IEEE Trans. Veh. Technol., vol. 58, no. 8, pp. 4645-4649, Oct. 2009.

[8] T. Ait-Idir, H. Chafnaji, and S. Saoudi, "Turbo packet combining for broadband space-time BICM hybrid-ARQ systems with co-channel interference," IEEE Trans. Wireless Commun., vol. 9, no. 5, pp. 16861697, May 2010.

[9] A. Rácz, N. Reider, and G. Fodor, "On the impact of inter-cell interference in LTE," in Proc. IEEE Globecom, Nov. 2008.

[10] H. Shirani-Mehr, H. Papadopoulos, S. A. Ramprashad, and G. Caire, "Joint scheduling and hybrid-ARQ for MU-MIMO downlink in the presence of inter-cell interference," IEEE Trans. Commun., vol. 59, no. 2, pp. 578-589, Feb. 2010.

[11] P. Viswanath, D. Tse, and R. Laroia, "Opportunistic beamforming using dumb antennas," IEEE Trans. Inform. Theory, vol. 6, no. 48, pp. 12771294, June 2002.

[12] J. Gil-Pelaez, "Note on the inversion theorem," Biometrika, vol. 38, no. 3-4, pp. 481-482, Dec. 1951.

[13] V. Witkovsky, "Computing the distribution of a linear combination of inverted gamma variables," Kybernetika, vol. 37, no. 1, pp. 79-90, 2001.

[14] W. Choi and J. G. Andrews, "Downlink performance and capacity of distributed antenna systems in a multicell environment," IEEE Trans. Wireless Commun., vol. 6, no. 1, pp. 69-73, Jan. 2007. 